\title{
Apps erobern auch die Medizin
}

\author{
Praktisch jeder, der ein Smartphone \\ hat, hat auch Apps. Diese mobilen \\ Programme für Handys zeichnen sich \\ nicht nur durch ihren Unterhaltungs- \\ wert aus, sondern können auch den \\ ärztlichen Alltag erleichtern.
}

- Mobile Applikationen - so genannte Apps - gehören zu den Hauptattraktionen von Smartphones wie dem iPhone oder von Handys mit Android-Betriebssystem. Diese kleinen Programme kann man aus dem Internet auf das Handy laden und direkt nach der Installation anwenden. Für den Gesundheitssektor werden hohe Erwartungen in die künftige Anwendung von Apps gesetzt.

So erwarten $67 \%$ der Unternehmen im medizinischen Bereich laut einer internationalen Studie des Marktforschungsunternehmens reseach2guidance, dass Apps in den kommenden fünf Jahren Ärzte und medizinisches Fachpersonal zunehmend unterstützen werden. Gesundheits-Apps können die Patienten in ihren Anstreungungen für Prävention und Fitness unterstützen.

\section{Überwachung chronisch Kranker}

Ein wichtiges Einsatzgebiet für Apps könnte die Versorgung chronisch Kranker (z. B. Diabetiker) sein, heißt es in der Studie, die jüngst vom Bundesverband Informationswirtschaft, Telekommunikation und neue Medien e. V. (BITKOM) vorgestellt wurde.

Inzwischen stehen in Deutschland bereits 1400 „Health-Apps“ zur Verfügung, international sind es über 7000 . Anders als in Deutschland werden in den skandinavischen Ländern und in den USA Gesundheits-Apps schon im medizinischen Alltag eingesetzt, berichtet Peter Ernste vom Gelsenkirchener Institut für Arbeit und Technik.

Natürlich steht auch hierzulande eine Reihe nützlicher Apps in den Startlöchern, z. B. die „CGM App“, mit der sich der Arzt bei seinen Haus- und Heimbesuchen Patientendaten aus dem Praxissystem auf dem iPhone anzeigen lassen kann. Die App ist von dem SoftwareHaus TurboMed entwickelt worden und soll in den nächsten Monaten auf den Markt kommen. Sie ist für alle Praxen nutzbar, die online sind und über ein Netz-Konto bei TurboMed verfügen.

\section{Patientendaten einfach unterwegs auf das Handy holen}

Mit Hilfe der kostenfreien Anwendung können Ärzte die Inhalte der jeweiligen Patientenkartei auf dem Handy abrufen. „Dabei werden die Daten nicht auf dem iPhone gespeichert", sagt Jens-Uwe Bäker von TurboMed. „Sobald die Anwendung wieder geschlossen wird, sind die Informationen nicht mehr abrufbar."

Bereits auf dem Markt ist die Applikation „iStethoscope“. Die App wurde am ComputerInstitut des University College in London entwickelt. Nach Auskunft des Entwicklers Peter Bentley wurde die 79
Cent teure Anwendung bereits mehrere Millionen Mal herunter geladen und treffe durchaus auf Zuspruch aus den ärztlichen Reihen. So begeistert sich der amerikanische Kardiologe Glenn Nordehn: „Das ist das Beste, was im Bereich der medizinischen Ausrüstung in den letzten Jahren auf den Markt gekommen ist. Ähnliche Geräte mit dieser Qualität sind 3000mal teurer."

Die Applikation verwandelt das iPhone in ein medizinisches Diagnosewerkzeug: Sie zeichnet Herzgeräusche auf. Dazu startet man das Programm und platziert das im iPhone eingebaute Mikrofon auf der nackten Brust. Allerdings räumt Bentley ein, dass er auch hin und wieder negative Rückmeldung erhalte, da die Handhabung ein gewisses Training erfordere.

\section{Fehlende Standardisierung, unklare Datensicherheit}

Trotz der Begeisterung für die schöne neue Gesundheitswelt gibt es auch skeptische Stimmen. Viele Gesundheits-Unternehmen beklagen in der research2guidane-Studie den Mangel an Standardisierung auf dem Smartphone-Markt.

Oftmals auch noch ungeklärt ist die Frage der Datensicherheit.

$\mathrm{GIA} / \mathrm{EB}$ = 